\title{
Algebraic and combinatorial structures on Baxter permutations
}

\author{
Samuele Giraudo ${ }^{1}$ \\ ${ }^{1}$ Institut Gaspard Monge, Université Paris-Est Marne-la-Vallée, Marne-la-Vallée, France
}

\begin{abstract}
We give a new construction of a Hopf subalgebra of the Hopf algebra of Free quasi-symmetric functions whose bases are indexed by objects belonging to the Baxter combinatorial family (i.e. Baxter permutations, pairs of twin binary trees, etc.). This construction relies on the definition of the Baxter monoid, analog of the plactic monoid and the sylvester monoid, and on a Robinson-Schensted-like insertion algorithm. The algebraic properties of this Hopf algebra are studied. This Hopf algebra appeared for the first time in the work of Reading [Lattice congruences, fans and Hopf algebras, Journal of Combinatorial Theory Series A, 110:237-273, 2005].

Résumé. Nous proposons une nouvelle construction d'une sous-algèbre de Hopf de l'algèbre de Hopf des fonctions quasi-symétriques libres dont les bases sont indexées par les objets de la famille combinatoire de Baxter (i.e. permutations de Baxter, couples d'arbres binaires jumeaux, etc.). Cette construction repose sur la définition du monoïde de Baxter, analogue du monoïde plaxique et du monoïde sylvestre, et d'un algorithme d'insertion analogue à l'algorithme de Robinson-Schensted. Les propriétés algébriques de cette algèbre de Hopf sont étudiées. Cette algèbre de Hopf est apparue pour la première fois dans le travail de Reading [Lattice congruences, fans and Hopf algebras, Journal of Combinatorial Theory Series A, 110:237-273, 2005].
\end{abstract}

Keywords: Hopf algebras, Robinson-Schensted algorithm, quotient monoid, Baxter permutations

\section{Introduction}

In the recent years, many combinatorial Hopf algebras, whose bases are indexed by combinatorial objects, have been intensively studied. For example, the Malvenuto-Reutenauer Hopf algebra FQSym of Free quasi-symmetric functions [19, 7] has bases indexed by permutations. This Hopf algebra admits several Hopf subalgebras: The Hopf algebra of Free symmetric functions FSym [21, 7], whose bases are indexed by standard Young tableaux, the Hopf algebra Bell [23] whose bases are indexed by set partitions, the Loday-Ronco Hopf algebra PBT [18, 12] whose bases are indexed by planar binary trees and the Hopf algebra Sym of non-commutative symmetric functions [10] whose bases are indexed by integer compositions. An unifying approach to construct all these structures relies on a definition of a congruence on words leading to the definition of monoids on combinatorial objects. Indeed, FSym is directly obtained from the plactic monoid [15], Bell from the Bell monoid [23], PBT from the sylvester monoid [11, 12], and Sym from the hypoplactic monoid [20]. The richness of these constructions relies on the fact that, in addition to construct Hopf algebras, the definition of such monoids often brings partial orders, combinatorial algorithms and Robinson-Schensted-like algorithms, of independent interest.

In this paper, we propose to enrich this collection of Hopf algebras by providing a construction of a Hopf algebra whose bases are indexed by objects belonging to the Baxter combinatorial family. This combinatorial family admits various representations as Baxter permutations [4], pairs of twin binary trees [8], 
quadrangulations [1], plane bipolar orientations [5], etc. In [22], Reading defines first a Hopf algebra on Baxter permutations in the context of lattice congruences; Moreover, very recently, Law and Reading [16] have studied and detailed their construction of this Hopf algebra. However, even if both points of view lead to the same general theory, their paths are different and provide different ways of understanding this Hopf algebra, one centered, as in Law and Reading's work, on lattice theory, the other, as in our work, centered on combinatorics on words. Moreover, a large part of the results of each paper does not appear in the other.

We begin by recalling in Section 2 the preliminary notions used thereafter. In Section 3 , we define the Baxter congruence. This congruence allows to define a quotient of the free monoid, the Baxter monoid, which has a number of properties required for the Hopf algebraic construction which follows. We show that the Baxter monoid is intimately linked to the sylvester monoid. Next, in Section 4, we develop a Robinson-Schensted-like insertion algorithm that allows to decide if two words are equivalent according to the Baxter congruence. Given a word, this algorithm computes a pair of twin binary trees. Section 5 is devoted to the study of some properties of the equivalence classes of permutations under the Baxter congruence. This leads to the definition of a lattice structure on pairs of twin binary trees. Finally, in Section 6, we define the Hopf algebra Baxter and study it. Using the order structure on pairs of twin binary trees, we provide multiplicative bases and show that Baxter is free as an algebra. Using the results of Foissy on bidendriform bialgebras [9], we show that Baxter is also self-dual and that the Lie algebra of its primitive elements is free.

\section{Acknowledgements}

The author would like to thank Florent Hivert and Jean-Christophe Novelli for their advice and help during all stages of the preparation of this paper. The computations of this work have been done with the open-source mathematical software Sage [25].

\section{Preliminaries}

\subsection{Words}

In the sequel, $A:=\left\{a_{1}<a_{2}<\ldots\right\}$ is a totally ordered infinite alphabet and $A^{*}$ is the free monoid spanned by $A$. Let $u \in A^{*}$. For $S \subseteq A$, we denote by $u_{\mid S}$ the restriction of $u$ on the alphabet $S$, that is the longest subword of $u$ made of letters of $S$. The evaluation eval $(u)$ of the word $u$ is the non-negative integer vector such that its $i$-th entry is the number of occurrences of the letter $a_{i}$ in $u$. Let $\max (u)$ be the maximal letter of $u$. The Schützenberger transformation \# is defined by $u^{\#}:=\max (u)+1-u_{|u|} \ldots \max (u)+1-u_{1}$; For example, $\left(a_{5} a_{3} a_{1} a_{1} a_{5} a_{2}\right)^{\#}=a_{4} a_{1} a_{5} a_{5} a_{3} a_{1}$. Note that it is an involution if $u$ has an occurrence of $a_{1}$. Let $v \in A^{*}$ and $\mathrm{a}, \mathrm{b} \in A$. The shuffle product $\amalg$ is defined on $\mathbb{Z}\langle A\rangle$ recursively by $u \amalg \epsilon:=\epsilon \amalg u:=u$ and $\mathrm{a} u \amalg \mathrm{b} v:=\mathrm{a}(u \amalg \mathrm{b} v)+\mathrm{b}(\mathrm{a} u \amalg v)$.

\subsection{Permutations}

Denote by $\mathfrak{S}_{n}$ the set of permutations of size $n$ and $\mathfrak{S}:=\cup_{n \geq 0} \mathfrak{S}_{n}$. We shall call $(i, j)$ a co-inversion of $\sigma \in \mathfrak{S}$ if $i<j$ and $\sigma_{i}^{-1}>\sigma_{j}^{-1}$. Let us recall that the (right) permutohedron order is the partial order $\leq_{\mathrm{P}}$ defined on $\mathfrak{S}_{n}$ where $\sigma$ is covered by $\nu$ if $\sigma=u \mathrm{ab} v$ and $\nu=u$ ba $v$ where a $<\mathrm{b}$. Let $\sigma, \nu \in \mathfrak{S}$. The permutation $\sigma / \nu$ is obtained by concatenating $\sigma$ and the letters of $\nu$ incremented by $|\sigma|$; In the same way, the permutation $\sigma \backslash \nu$ is obtained by concatenating the letters of $\nu$ incremented by $|\sigma|$ and $\sigma$; For example, 312 $/ 2314=\mathbf{3 1 2 5 6 4 7}$ and $\mathbf{3 1 2} \backslash 2314=5647312$. The permutation $\sigma$ is connected if $\sigma=\nu / \pi$ implies $\nu=\sigma$ or $\pi=\sigma$. The shifted shuffle product $\bar{\varpi}$ of two permutations is defined by $\sigma \bar{\varpi} \nu:=$ 
$\sigma \amalg\left(\nu_{1}+|\sigma| \ldots \nu_{|\nu|}+|\sigma|\right)$; For example, 12Ш $21=\mathbf{1 2} \amalg 43=\mathbf{1 2} 43+\mathbf{1 4 2 3}+\mathbf{1 4 4 2}+4 \mathbf{1 2 3}+4 \mathbf{1 3 2}+43 \mathbf{1 2}$. The standardized word $\operatorname{std}(u)$ of $u \in A^{*}$ is the unique permutation $\sigma$ satisfying $\sigma_{i}<\sigma_{j}$ iff $u_{i} \leq u_{j}$ for all $1 \leq i<j \leq|u|$; For example, $\operatorname{std}\left(a_{3} a_{1} a_{4} a_{2} a_{5} a_{7} a_{4} a_{2} a_{3}\right)=416289735$.

\subsection{Binary trees}

Denote by $\mathcal{B} \mathcal{T}_{n}$ the set of binary trees with $n$ internal nodes and $\mathcal{B T}:=\cup_{n \geq 0} \mathcal{B T}_{n}$. We use in the sequel the standard terminology (i.e., child, ancestor, ...) about binary trees [2]. The only element of $\mathcal{B} \mathcal{T}_{0}$ is the leaf or empty tree, denoted by $\perp$. Let us recall that the Tamari order [14] is the partial order $\leq_{\mathrm{T}}$ defined on $\mathcal{B} \mathcal{T}_{n}$ where $T_{0} \in \mathcal{B} \mathcal{T}_{n}$ is covered by $T_{1} \in \mathcal{B} \mathcal{T}_{n}$ if it is possible to transform $T_{0}$ into $T_{1}$ by performing a right rotation (see Figure 1 .

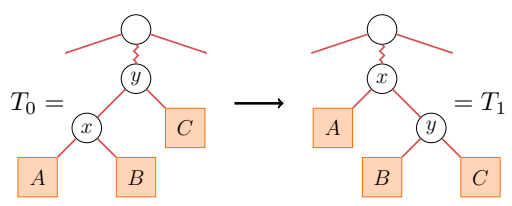

Figure 1: The right rotation of root $y$.

Let $T_{0}, T_{1} \in \mathcal{B T}$. The binary tree $T_{0} / T_{1}$ is obtained by grafting $T_{0}$ from its root on the leftmost leaf of $T_{1}$; In the same way, the binary tree $T_{0} \backslash T_{1}$ is obtained by grafting $T_{1}$ from its root on the rightmost leaf of $T_{0}$. The canopy (see [18] and [26]) $\operatorname{cnp}(T)$ of $T \in \mathcal{B T}$ is the word on the alphabet $\{0,1\}$ obtained by browsing the leaves of $T$ from left to right except the first and the last one, writing 0 if the considered leaf is oriented to the right, 1 otherwise (see Figure 2). Note that the orientation of the leaves in a binary tree is determined only by its nodes so that we can omit to draw the leaves in our next graphical representations.

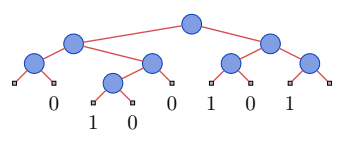

Figure 2: The canopy of this binary tree is 0100101 .

An $A$-labeled binary tree $T$ is a left (resp. right) binary search tree if for any node $x$ labeled by b, each label a of a node in the left subtree of $x$ and each label c of a node in the right subtree of $x$, the inequality $\mathrm{a}<\mathrm{b} \leq \mathrm{c}$ (resp. $\mathrm{a} \leq \mathrm{b}<\mathrm{c}$ ) holds. A binary tree $T \in \mathcal{B} \mathcal{T}_{n}$ is a decreasing binary tree if it is bijectively labeled on $\{1, \ldots, n\}$ and, for all node $y$ of $T$, if $x$ is a child of $y$, then the label of $x$ is smaller than the label of $y$. The shape of a labeled binary tree is the unlabeled binary tree obtained by forgetting its labels.

\subsection{Baxter permutations and pairs of twin binary trees}

A permutation $\sigma$ is a Baxter permutation if for any subword $u=u_{1} u_{2} u_{3} u_{4}$ of $\sigma$ such that the letters $u_{2}$ and $u_{3}$ are adjacent in $\sigma, \operatorname{std}(u) \notin\{2413,3142\}$. In other words, $\sigma$ is a Baxter permutation if it avoids the generalized permutation patterns $2-41-3$ and $3-14-2$ (see [3] for an introduction on generalized permutation patterns). For example, 42173856 is not a Baxter permutation; On the other hand 436975128 is a Baxter permutation. Let us denote by $\mathfrak{S}_{n}^{\mathrm{B}}$ the set of Baxter permutations of size $n$ and $\mathfrak{S}^{\mathrm{B}}:=\cup_{n \geq 0} \mathfrak{S}_{n}^{\mathrm{B}}$.

A pair of twin binary trees $\left(T_{L}, T_{R}\right)$ is made of two binary trees $T_{L}, T_{R} \in \mathcal{B} \mathcal{T}_{n}$ such that the canopies of $T_{L}$ and $T_{R}$ are complementary, that is $\operatorname{cnp}\left(T_{L}\right)_{i} \neq \operatorname{cnp}\left(T_{R}\right)_{i}$ for all $1 \leq i \leq n-1$. Denote by $\mathcal{T} \mathcal{B} \mathcal{T}_{n}$ 
the set of pairs of twin binary trees where each binary tree has $n$ nodes and $\mathcal{T B} \mathcal{T}:=\cup_{n \geq 0} \mathcal{T} \mathcal{B} \mathcal{T}_{n}$. In [8], Dulucq and Guibert have highlighted a bijection between Baxter permutations and pairs of twin binary trees. In the sequel, we shall make use of a very similar bijection.

\section{The Baxter monoid}

\subsection{Definition and first properties}

Recall that an equivalence relation $\equiv$ defined on $A^{*}$ is a congruence if for all $u, u^{\prime}, v, v^{\prime} \in A^{*}, u \equiv u^{\prime}$ and $v \equiv v^{\prime}$ imply $u . v \equiv u^{\prime} \cdot v^{\prime}$.

Definition 3.1 The Baxter monoid is the quotient of the free monoid $A^{*}$ by the congruence $\equiv_{\mathrm{B}}$ that is the transitive closure of the adjacency relations $\leftrightharpoons_{\mathrm{B}}$ and $\rightleftharpoons_{\mathrm{B}}$ defined for $u, v \in A^{*}$ and $\mathrm{a}, \mathrm{b}, \mathrm{c}, \mathrm{d} \in A$ by:

$$
\begin{aligned}
& \mathrm{c} u \mathrm{ad} v \mathrm{~b} \leftrightharpoons \mathrm{B} \mathrm{c} u \mathrm{da} v \mathrm{~b} \text { where } \mathrm{a} \leq \mathrm{b}<\mathrm{c} \leq \mathrm{d} \text {, } \\
& \mathrm{b} u \mathrm{da} v \mathrm{c} \rightleftharpoons{ }_{\mathrm{B}} \mathrm{b} u \mathrm{ad} v \mathrm{c} \quad \text { where } \mathrm{a}<\mathrm{b} \leq \mathrm{c}<\mathrm{d} \text {. }
\end{aligned}
$$

For $u \in A^{*}$, denote by $\widehat{u}$ the $\equiv_{\mathrm{B}}$-equivalence class of $u$; For example, the $\equiv_{\mathrm{B}}$-equivalence class of 5273641 is $\{5237641,5273641,5276341,5723641,5726341,5762341\}$.

An equivalence relation $\equiv$ defined on $A^{*}$ is compatible with the restriction of alphabet intervals if for all interval $I$ of $A$ and for all $u, v \in A^{*}, u \equiv v$ implies $u_{\mid I} \equiv v_{\mid I}$.

Proposition 3.2 The Baxter monoid is compatible with the restriction of alphabet intervals.

Proof: We only have to check the property on adjacency relations.

An equivalence relation $\equiv$ defined on $A^{*}$ is compatible with the destandardization process if for all $u, v \in A^{*}, u \equiv v$ iff $\operatorname{std}(u) \equiv \operatorname{std}(v)$ and $\operatorname{eval}(u)=\operatorname{eval}(v)$.

Proposition 3.3 The Baxter monoid is compatible with the destandardization process.

An equivalence relation $\equiv$ defined on $A^{*}$ is compatible with the Schützenberger involution if for all $u, v \in A^{*}, u \equiv v$ implies $u^{\#} \equiv v^{\#}$.

Proposition 3.4 The Baxter monoid is compatible with the Schützenberger involution.

\subsection{Connection with the sylvester monoid}

The sylvester monoid [11, 12] is the quotient of the free monoid $A^{*}$ by the congruence $\equiv_{\mathrm{S}}$ that is the transitive closure of the adjacency relation $\leftrightharpoons_{\mathrm{S}}$ defined for $u \in A^{*}$ and $\mathrm{a}, \mathrm{b}, \mathrm{c} \in A$ by:

$$
\mathrm{ac} u \mathrm{~b} \leftrightharpoons_{\mathrm{S}} \mathrm{ca} u \mathrm{~b} \quad \text { where } \mathrm{a} \leq \mathrm{b}<\mathrm{c} .
$$

In the same way, let us define the \#-sylvester monoid by the congruence $\equiv_{\mathrm{S} \#}$ that is the transitive closure of the adjacency relation $\leftrightharpoons_{\mathrm{S} \#}$ defined for $u \in A^{*}$ and $\mathrm{a}, \mathrm{b}, \mathrm{c} \in A$ by:

$$
\mathrm{b} u \mathrm{ac} \leftrightharpoons \mathrm{s} \# \mathrm{~b} u \mathrm{ca} \quad \text { where } \mathrm{a}<\mathrm{b} \leq \mathrm{c} .
$$

Note that this adjacency relation is defined by taking the images by the Schützenberger involution of the sylvester adjacency relation. Indeed, for all $u, v \in A^{*}, u \equiv_{\mathrm{S} \#} v$ iff $u^{\#} \equiv_{\mathrm{S}} v^{\#}$. The Baxter monoid and the sylvester monoid are related in the following way:

Proposition 3.5 Let $u, v \in A^{*}$. Then, $u \equiv_{\mathrm{B}} v$ iff $u \equiv_{\mathrm{S}} v$ and $u \equiv_{\mathrm{S} \#} v$.

Proposition 3.5 shows that the $\equiv_{\mathrm{B}}$-equivalence classes are the intersection of $\equiv_{\mathrm{S}}$-equivalence classes and $\equiv_{\mathrm{S} \#}$-equivalence classes. 


\section{A Robinson-Schensted-like algorithm}

We shall describe here an insertion algorithm $u \mapsto(\mathbb{P}(u), \mathbb{Q}(u))$, such that, given a word $u \in A^{*}$, it computes its $\mathbb{P}$-symbol, that is a pair of $A$-labeled twin binary trees $\left(T_{L}, T_{R}\right)$ where $T_{L}$ (resp. $\left.T_{R}\right)$ is a left (resp. right) binary search tree, and its $\mathbb{Q}$-symbol, a decreasing binary tree.

\subsection{Definition of the insertion algorithm}

Let $T$ be an $A$-labeled right binary search tree and b a letter of $A$. The lower restricted binary tree of $T$ compared to $\mathrm{b}$, namely $T_{\leq \mathrm{b}}$, is the right binary search tree uniquely made of the nodes $x$ of $T$ labeled by a letter a satisfying $\mathrm{a} \leq \mathrm{b}$ and such that for all nodes $x$ and $y$ of $T_{\leq \mathrm{b}}$, if $x$ is ancestor of $y$ in $T_{\leq \mathrm{b}}$, then $x$ is ancestor of $y$ in $T$. In the same way, we define the higher restricted binary tree of $T$ compared to $\mathrm{b}$, namely $T_{>\mathrm{b}}$ (see Figure 3 ).
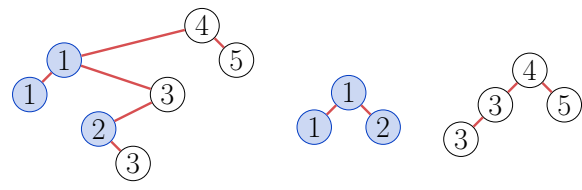

Figure 3: A right binary search tree $T, T_{\leq 2}$ and $T_{>2}$.

Let $T$ be an $A$-labeled right binary search tree and a a letter of $A$. The root insertion of a into $T$ consists in modifying $T$ so that the root of $T$ is a new node labeled by a, its left subtree is $T_{\leq \mathrm{a}}$ and its right subtree is $T_{>\mathrm{a}}$.

Let $T$ be an $A$-labeled left (resp. right) binary search tree and a a letter of $A$. The leaf insertion of a into $T$ is recursively defined by: If $T=\perp$, the result is the one-node binary tree labeled by a; Else, if the label $\mathrm{b}$ of the root of $T$ satisfies $\mathrm{a}<\mathrm{b}$ (resp. $\mathrm{a} \leq \mathrm{b}$ ), make a leaf insertion of a into the left subtree of $T$, else, make a leaf insertion of a into the right subtree of $T$.

Given a pair of $A$-labeled twin binary trees $\left(T_{L}, T_{R}\right)$ where $T_{L}$ (resp. $\left.T_{R}\right)$ is a left (resp. right) binary search tree, the insertion of the letter a of $A$ into $\left(T_{L}, T_{R}\right)$ consists in making a leaf insertion of a into $T_{L}$ and a root insertion of a into $T_{R}$.

The $\mathbb{P}$-symbol $\left(T_{L}, T_{R}\right)$ of a word $u \in A^{*}$ is computed by iteratively inserting the letters of $u$, from left to right, into the pair of twin binary trees $(\perp, \perp)$. The $\mathbb{Q}$-symbol of $u$ is the decreasing binary tree labeled on $\{1, \ldots,|u|\}$, built by recording the dates of creation of each node of $T_{R}$ (see Figure 4 .
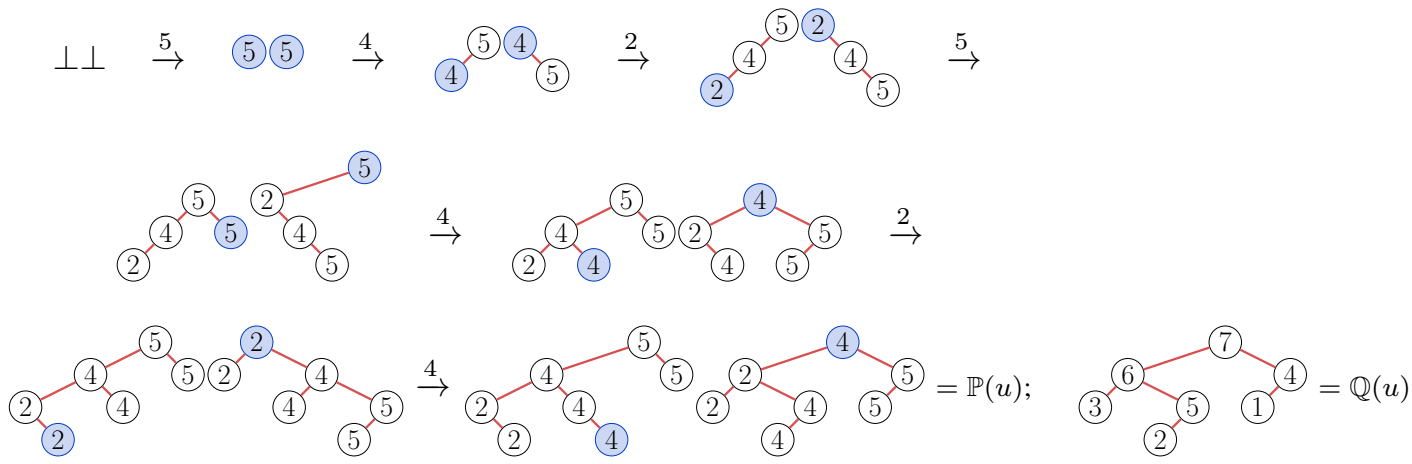

Figure 4: Steps of computation of the $\mathbb{P}$-symbol and the $\mathbb{Q}$-symbol of $u:=5425424$. 


\subsection{Validity of the insertion algorithm}

Lemma 4.1 Let $u \in A^{*}$. Let $T$ be the right binary search tree obtained by root insertions of the letters of $u$, from left to right. Let $T^{\prime}$ be the right binary search tree obtained by leaf insertions of the letters of $u$, from right to left. Then, $T=T^{\prime}$.

Lemma 4.2 Let $\sigma \in \mathfrak{S}$ and $T \in \mathcal{B} \mathcal{T}_{|\sigma|}$ be the binary search tree obtained by leaf insertions of the letters of $\sigma$, from left to right. Then, for $1 \leq i \leq|\sigma|-1$, the $i+1$-st leaf of $T$ is right-oriented iff $(i, i+1)$ is a co-inversion of $\sigma$.

If $\left(T_{L}, T_{R}\right)$ is a pair of labeled twin binary trees, define its shape, that is the pair of unlabeled twin binary trees $\left(T_{L}^{\prime}, T_{R}^{\prime}\right)$ where $T_{L}^{\prime}$ (resp. $T_{R}^{\prime}$ ) is the shape of $T_{L}$ (resp. $T_{R}$ ).

Proposition 4.3 For all word $u \in A^{*}$, the shape of the $\mathbb{P}$-symbol of $u$ is a pair of twin binary trees.

Proposition 4.4 Let $u, v \in A^{*}$. Then, $u \equiv_{\mathrm{B}} v$ iff $\mathbb{P}(u)=\mathbb{P}(v)$.

In particular, we have $\mathbb{P}(\sigma)=\mathbb{P}(\nu)$ iff the permutations $\sigma$ and $\nu$ are $\equiv_{\mathrm{B}}$-equivalent. Moreover, each $\equiv_{\mathrm{B}}$-equivalence class of permutations can be encoded by a pair of unlabeled twin binary trees because there is one unique way to bijectively label a binary tree with $n$ nodes on $\{1, \ldots, n\}$ such that it is a binary search tree.

Remark 4.5 Let $u, v \in A^{*}$ and $\left(T_{L}, T_{R}\right):=\mathbb{P}(u)$. We have $u \equiv_{\mathrm{B}} v$ iff the following two assertions are satisfied:

(i) $v$ is a linear extension of $T_{L}$ seen as a poset in which the smallest element is its root;

(ii) $v$ is a linear extension of $T_{R}$ seen as a poset in which minimal elements are the nodes with no descents.

\section{The Baxter lattice}

\subsection{Some properties of the $\equiv_{\mathrm{B}}$-equivalence classes of permutations}

Theorem 5.1 For all $n \geq 0$, each equivalence class of $\mathfrak{S}_{n} / \equiv_{B}$ contains exactly one Baxter permutation.

Proposition 5.2 For all $n \geq 0$, each equivalence class of $\mathfrak{S}_{n} / \equiv_{B}$ is an interval of the permutohedron.

For all permutation $\sigma$, let us define $\sigma \uparrow$ (resp. $\sigma \downarrow$ ) the maximal (resp. minimal) permutation of the $\equiv_{\mathrm{B}}$-equivalence class of $\sigma$ for the permutohedron order.

Proposition 5.3 Let $\sigma, \nu \in \mathfrak{S}_{n}$ such that $\sigma \leq_{\mathrm{P}} \nu$. Then, $\sigma \uparrow \leq_{\mathrm{P}} \nu \uparrow$ and $\sigma \downarrow \leq_{\mathrm{P}} \nu \downarrow$.

\subsection{A lattice structure on the set of pairs of twin binary trees}

Definition 5.4 For all $n \geq 0$, define the order relation $\leq_{\mathrm{B}}$ on the set $\mathcal{T} \mathcal{B} \mathcal{T}_{n}$ setting $J_{0} \leq_{\mathrm{B}} J_{1}$, where $J_{0}, J_{1} \in \mathcal{T} \mathcal{B} \mathcal{T}_{n}$, if there exists $\sigma_{0}, \sigma_{1} \in \mathfrak{S}_{n}$ such that $\mathbb{P}\left(\sigma_{0}\right)=J_{0}, \mathbb{P}\left(\sigma_{1}\right)=J_{1}$ and $\sigma_{0} \leq_{\mathrm{P}} \sigma_{1}$.

Propositions 5.2 and 5.3 ensure that this order is well-defined, and in particular that the relation $\leq_{\mathrm{B}}$ is transitive and antisymmetric.

The pair of twin binary trees $\left(T_{L}, T_{R}\right)$ is covered by $\left(T_{L}^{\prime}, T_{R}^{\prime}\right) \in \mathcal{T} \mathcal{B} \mathcal{T}$ if one of the three following conditions is satisfied:

1. $T_{R}^{\prime}=T_{R}$ and $T_{L}^{\prime}$ is obtained from $T_{L}$ by performing a left rotation into $T_{L}$ such that $\operatorname{cnp}\left(T_{L}\right)=$ $\operatorname{cnp}\left(T_{L}^{\prime}\right)$ 
2. $T_{L}^{\prime}=T_{L}$ and $T_{R}^{\prime}$ is obtained from $T_{R}$ by performing a right rotation into $T_{R}$ such that $\operatorname{cnp}\left(T_{R}\right)=$ $\operatorname{cnp}\left(T_{R}^{\prime}\right)$;

3. $T_{L}^{\prime}$ (resp. $T_{R}^{\prime}$ ) is obtained by performing a left (resp. right) rotation into $T_{L}$ (resp. $T_{R}$ ) such that $\operatorname{cnp}\left(T_{L}\right) \neq \operatorname{cnp}\left(T_{L}^{\prime}\right)\left(\right.$ resp. $\left.\operatorname{cnp}\left(T_{R}\right) \neq \operatorname{cnp}\left(T_{R}^{\prime}\right)\right)$.

Moreover, it is possible to compare two pairs of twin binary trees $J_{0}:=\left(T_{L}^{0}, T_{R}^{0}\right)$ and $J_{1}:=\left(T_{L}^{1}, T_{R}^{1}\right)$ very easily by computing the Tamari vector (see [14]) of each binary tree. Indeed, we have $J_{0} \leq_{\mathrm{B}} J_{1}$ iff the Tamari vector of $T_{L}^{0}$ (resp. $T_{R}^{0}$ ) is greater (resp. smaller) component by component than the Tamari vector of $T_{L}^{1}$ (resp. $T_{R}^{1}$ ).

Propositions 5.2 and 5.3 implies that that $\equiv_{\mathrm{B}}$ is also a lattice congruence [6, 22]. Thus, since the permutohedron is a lattice,

Proposition 5.5 For all $n \geq 0$, the poset $\left(\mathcal{T} \mathcal{B} \mathcal{T}_{n}, \leq_{\mathrm{B}}\right)$ is a lattice.

\section{The Baxter Hopf Algebra}

In the sequel, all the algebraic structures have a field of characteristic zero $\mathbb{K}$ as ground field.

\subsection{The Hopf algebra FQSym}

Recall that the family $\left\{\mathbf{F}_{\sigma}\right\}_{\sigma \in \mathfrak{S}}$ form the fundamental basis of FQSym [7]. Its product and its coproduct are defined by:

$$
\mathbf{F}_{\sigma} \cdot \mathbf{F}_{\nu}:=\sum_{\pi \in \sigma \varpi \nu} \mathbf{F}_{\pi}, \quad \Delta\left(\mathbf{F}_{\sigma}\right):=\sum_{0 \leq i \leq|\sigma|} \mathbf{F}_{\operatorname{std}\left(\sigma_{1} \ldots \sigma_{i}\right)} \otimes \mathbf{F}_{\operatorname{std}\left(\sigma_{i+1} \ldots \sigma_{|\sigma|}\right)} .
$$

The following theorem due to Hivert and Nzeutchap [13] shows that an equivalence relation on $A^{*}$ satisfying some properties can be used to define Hopf subalgebras of FQSym:

Theorem 6.1 Let $\equiv$ be an equivalence relation defined on $A^{*}$. If $\equiv$ is a congruence, compatible with the restriction of alphabet intervals and compatible with the destandardization process, then, the family $\left\{\mathbf{P}_{\widehat{\sigma}}\right\}_{\widehat{\sigma} \in \mathfrak{S} / \equiv}$ defined by:

$$
\mathbf{P}_{\widehat{\sigma}}:=\sum_{\sigma \in \widehat{\sigma}} \mathbf{F}_{\sigma}
$$

spans a Hopf subalgebra of FQSym.

\subsection{The Hopf algebra Baxter}

By definition, $\equiv_{\mathrm{B}}$ is a congruence, and, by Proposition 3.2 and 3.3 . $\equiv_{\mathrm{B}}$ checks the conditions of Theorem 6.1 Moreover, by Proposition 4.4 the $\equiv_{\mathrm{B}}$-equivalence classes of permutations can be encoded by pairs of unlabeled twin binary trees. Hence, we have the following theorem:

Theorem 6.2 The family $\left\{\mathbf{P}_{J}\right\}_{J \in \mathcal{T} \mathcal{B} \mathcal{T}}$ defined by:

$$
\mathbf{P}_{J}:=\sum_{\substack{\sigma \in \mathfrak{S} \\ \mathbb{P}(\sigma)=J}} \mathbf{F}_{\sigma}
$$

spans a Hopf subalgebra of FQSym, namely the Hopf algebra Baxter. 
The Hilbert series of Baxter is $B(z):=1+z+2 z^{2}+6 z^{3}+22 z^{4}+92 z^{5}+422 z^{6}+2074 z^{7}+$ $10754 z^{8}+58202 z^{9}+326240 z^{10}+1882960 z^{11}+\ldots$, the generating series of Baxter permutations (sequence A001181 of [24]).

One has for example,

$$
\mathbf{P}_{00^{\circ}}=\mathbf{F}_{12}, \quad \mathbf{P}_{2000}=\mathbf{F}_{2143}+\mathbf{F}_{2413}, \quad \mathbf{P}_{000}=\mathbf{F}_{542163}+\mathbf{F}_{542613}+\mathbf{F}_{546213}
$$

By Theorem 6.1, the product of Baxter is well-defined. We deduce it from the product of FQSym and obtain

$$
\mathbf{P}_{J_{0}} \cdot \mathbf{P}_{J_{1}}=\sum_{\substack{\mathbb{P}(\sigma)=J_{0}, \mathbb{P}(\nu)=J_{1} \\ \pi \in \sigma \bar{\amalg} \nu \cap \mathfrak{S}^{\mathrm{B}}}} \mathbf{P}_{\mathbb{P}(\pi)}
$$

For example,

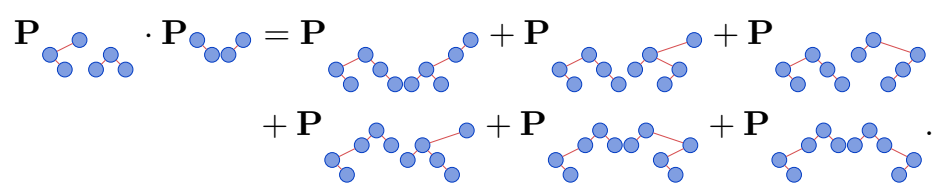

In the same way, we deduce the coproduct of Baxter from the coproduct of FQSym and obtain

$$
\Delta\left(\mathbf{P}_{J}\right)=\sum_{\substack{\mathbb{P}(\pi)=J \\ \pi=u \cdot v \\ \sigma:=\operatorname{std}(u), \nu:=\operatorname{std}(v) \in \mathfrak{S}^{\mathrm{B}}}} \mathbf{P}_{\mathbb{P}(\sigma)} \otimes \mathbf{P}_{\mathbb{P}(\nu)} .
$$

For example,

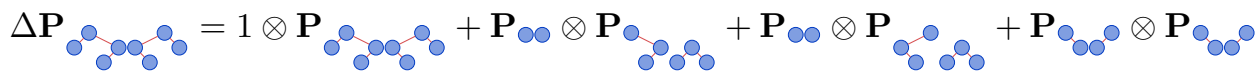

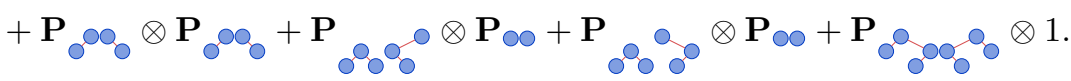

Remark 6.3 It is well-known that the Hopf algebra PBT [18, 12] is a Hopf subalgebra of FQSym. Besides, we have the following sequence of injective Hopf maps:

$$
\text { PBT } \stackrel{\rho}{\hookrightarrow} \text { Baxter } \hookrightarrow \text { FQSym. }
$$

Indeed, by Proposition 3.5. every $\equiv_{\mathrm{S}}$-equivalence class is an union of some $\equiv_{\mathrm{B}}$-equivalence classes. Denoting by $\left\{\mathbf{P}_{T}\right\}_{T \in \mathcal{B} \mathcal{T}}$ the basis of $\mathbf{P B T}$ defined in accordance with 6 by the sylvester equivalence relation $\equiv_{\mathrm{S}}$, we have

$$
\rho\left(\mathbf{P}_{T}\right)=\sum_{\substack{T^{\prime} \in \mathcal{B} \mathcal{T} \\ J:=\left(T^{\prime}, T\right) \in \mathcal{T} \mathcal{B} \mathcal{T}}} \mathbf{P}_{J}
$$

For example,

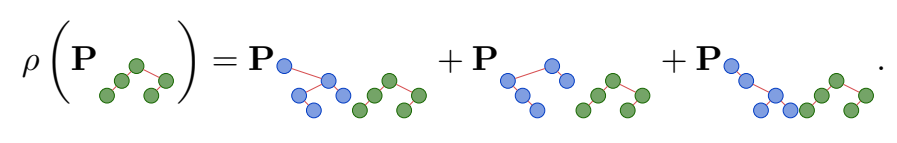




\subsection{Multiplicative bases}

Define the elementary family $\left\{\mathbf{E}_{J}\right\}_{J \in \mathcal{T} \mathcal{B} \mathcal{T}}$ and the homogeneous family $\left\{\mathbf{H}_{J}\right\}_{J \in \mathcal{T} \mathcal{B} \mathcal{T}}$ respectively by:

$$
\mathbf{E}_{J}:=\sum_{J \leq \mathrm{B}} \mathbf{P}_{J^{\prime}} \quad \text { and } \quad \mathbf{H}_{J}:=\sum_{J^{\prime} \leq_{\mathrm{B}} J} \mathbf{P}_{J^{\prime}} .
$$

These families are bases of Baxter since they are defined by triangularity.

Let $J_{0}:=\left(T_{L}^{0}, T_{R}^{0}\right)$ and $J_{1}:=\left(T_{L}^{1}, T_{R}^{1}\right)$ be two pairs of twin binary trees. Let us define the pair of twin binary trees $J_{0} / J_{1}$ by $J_{0} / J_{1}:=\left(T_{L}^{0} \backslash T_{L}^{1}, T_{R}^{0} / T_{R}^{1}\right)$. In the same way, the pair of twin binary trees $J_{0} \backslash J_{1}$ is defined by $J_{0} \backslash J_{1}:=\left(T_{L}^{0} / T_{L}^{1}, T_{R}^{0} \backslash T_{R}^{1}\right)$.

Using the multiplicative bases of FQSym, we establish the following proposition:

Proposition 6.4 For all $J_{0}, J_{1} \in \mathcal{T} \mathcal{B} \mathcal{T}$, we have

$$
\mathbf{E}_{J_{0}} \cdot \mathbf{E}_{J_{1}}=\mathbf{E}_{J_{0} / J_{1}} \quad \text { and } \quad \mathbf{H}_{J_{0}} \cdot \mathbf{H}_{J_{1}}=\mathbf{H}_{J_{0} \backslash J_{1}} .
$$

Lemma 6.5 Let $C$ be an equivalence class of $\mathfrak{S}_{n} / \equiv_{\mathrm{B}}$. The Baxter permutation belonging to $C$ is connected iff all the permutations of $C$ are connected.

Let us say that a pair of twin binary trees $J$ is connected if the unique Baxter permutation $\sigma$ satisfying $\mathbb{P}(\sigma)=J$ is connected.

Proposition 6.6 The Hopf algebra Baxter is free on the elements $\mathbf{E}_{J}$ where $J$ is a connected pair of twin binary trees.

The generating series $B_{C}(z)$ of connected Baxter permutations is $B_{C}(z)=1-B(z)^{-1}$. First dimensions of algebraic generators of Baxter are 1, 1, 1, 3, 11, 47, 221, 1113, 5903, 32607, 186143, 1092015 .

\subsection{Bidendriform bialgebra structure}

A Hopf algebra $(H, \cdot, \Delta)$ can be fitted into a bidendriform bialgebra structure [9] if $\left(H^{+}, \prec, \succ\right)$ is a dendriform algebra [17] and $\left(H^{+}, \Delta_{\prec}, \Delta_{\succ}\right)$ a codendriform coalgebra, where $H^{+}$is the augmentation ideal of $H$. The operators $\prec, \succ, \Delta_{\prec}$ and $\Delta_{\succ}$ have to fulfil some compatibility relations. In particular, for all $x, y \in H^{+}$, the product . of $H$ is retrieved by $x \cdot y=x \prec y+x \succ y$ and the coproduct $\Delta$ of $H$ is retrieved by $\Delta(x)=1 \otimes x+\Delta_{\prec}(x)+\Delta_{\succ}(x)+x \otimes 1$.

The Hopf algebra FQSym admits a bidendriform bialgebra structure [9]. Indeed, for all $\sigma, \nu \in \mathfrak{S}$ set

$$
\begin{aligned}
& \mathbf{F}_{\sigma} \prec \mathbf{F}_{\nu}:=\sum_{\substack{\pi \in \sigma \bar{W} \nu \\
\pi|\pi|=\sigma|\sigma|}} \mathbf{F}_{\pi}, \quad \mathbf{F}_{\sigma} \succ \mathbf{F}_{\nu}:=\sum_{\substack{\pi \in \sigma \varpi \nu \\
\pi_{|\pi|}=\nu_{|\nu|}+|\sigma|}} \mathbf{F}_{\pi}, \\
& \Delta_{\prec}\left(\mathbf{F}_{\sigma}\right):=\sum_{\sigma_{|\sigma|}^{-1} \leq i \leq|\sigma|-1} \mathbf{F}_{\operatorname{std}\left(\sigma_{1} \ldots \sigma_{i}\right)} \otimes \mathbf{F}_{\operatorname{std}\left(\sigma_{i+1} \ldots \sigma_{|\sigma|}\right)}, \\
& \Delta_{\succ}\left(\mathbf{F}_{\sigma}\right):=\sum_{1 \leq i \leq \sigma_{|\sigma|}^{-1}-1} \mathbf{F}_{\operatorname{std}\left(\sigma_{1} \ldots \sigma_{i}\right)} \otimes \mathbf{F}_{\operatorname{std}\left(\sigma_{i+1} \ldots \sigma_{|\sigma|}\right)} .
\end{aligned}
$$

Proposition 6.7 If $\equiv$ is an equivalence relation defined on $A^{*}$ satisfying the conditions of Theorem 6.1 and additionally, for all $u, v \in A^{*}$, the relation $u \equiv v$ implies $u_{|u|}=v_{|v|}$, then, the family defined in (6) spans a bidendriform sub-bialgebra of FQSym, and is free as an algebra, cofree as a coalgebra, self-dual, and the Lie algebra of its primitive elements is free.

The equivalence relation $\equiv_{\mathrm{B}}$ satisfies the premises of Proposition 6.7 so that Baxter is free as an algebra, cofree as a coalgebra, self-dual, and the Lie algebra of its primitive elements is free. 


\subsection{The dual Hopf algebra Baxter ${ }^{\star}$}

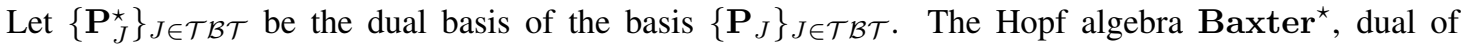
Baxter, is a quotient Hopf algebra of FQSym ${ }^{\star}$. More precisely,

$$
\text { Baxter }^{\star}=\text { FQSym }^{\star} / I
$$

where $I$ is the Hopf ideal of $\mathbf{F Q S y m}{ }^{\star}$ spanned by the relations $\mathbf{F}_{\sigma}^{\star}=\mathbf{F}_{\nu}^{\star}$ whenever $\sigma \equiv_{\mathrm{B}} \nu$.

Let $\phi: \mathbf{F Q S y m}^{\star} \rightarrow$ Baxter $^{\star}$ be the canonical projection, mapping $\mathbf{F}_{\sigma}^{\star}$ on $\mathbf{P}_{J}^{\star}$ whenever $\mathbb{P}(\sigma)=J$. By definition, the product of Baxter ${ }^{\star}$ is

$$
\mathbf{P}_{J_{0}}^{\star} \cdot \mathbf{P}_{J_{1}}^{\star}=\phi\left(\mathbf{F}_{\sigma}^{\star} \cdot \mathbf{F}_{\nu}^{\star}\right)
$$

where $\sigma$ and $\nu$ are any permutations such that $\mathbb{P}(\sigma)=J_{0}$ and $\mathbb{P}(\nu)=J_{1}$. For example,

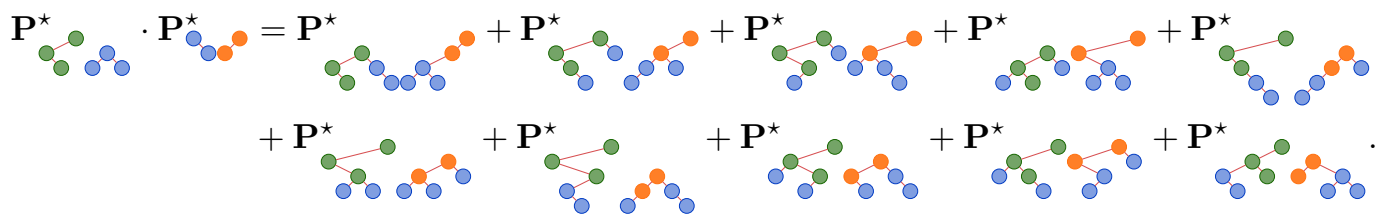

In the same way, the coproduct of Baxter ${ }^{\star}$ is

$$
\Delta\left(\mathbf{P}_{J}\right)=(\phi \otimes \phi)\left(\Delta\left(\mathbf{F}_{\sigma}^{\star}\right)\right)
$$

where $\sigma$ is any permutation such that $\mathbb{P}(\sigma)=J$. For example,

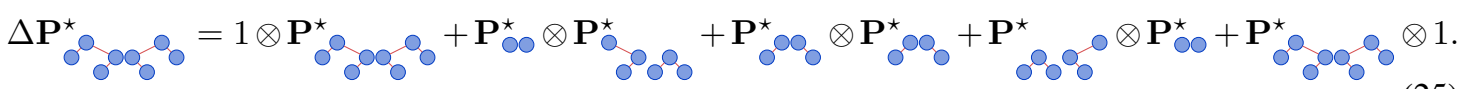

Remark 6.8 By Proposition 6.7 the Hopf algebras Baxter and Baxter $^{\star}$ are isomorphic. However, denoting by $\theta:$ Baxter $\hookrightarrow$ FQSym the injection from Baxter to FQSym, $\psi:$ FQSym $\leftrightarrow$ FQSym ${ }^{\star}$ the isomorphism from FQSym to $\mathbf{F Q S y m}^{\star}$ defined by $\psi\left(\mathbf{F}_{\sigma}\right):=\mathbf{F}_{\sigma^{-1}}^{\star}$, and $\phi: \mathbf{F Q S y m}^{\star} \rightarrow$ Baxter $^{\star}$ the surjection from FQSym $^{\star}$ to Baxter $^{\star}$, the map $\phi \circ \psi \circ \theta:$ Baxter $\rightarrow$ Baxter $^{\star}$ is not an isomorphism. Indeed:

$$
\begin{aligned}
& \phi \circ \psi \circ \theta \mathbf{P}_{000}=\phi \circ \psi\left(\mathbf{F}_{2143}+\mathbf{F}_{2413}\right)=\phi\left(\mathbf{F}_{2143}^{\star}+\mathbf{F}_{3142}^{\star}\right)=\mathbf{P}^{\star} \\
& \phi \circ \psi \circ \theta \mathbf{P}_{200}
\end{aligned}
$$

showing that $\phi \circ \psi \circ \theta$ is not injective.

\subsection{Primitive and totally primitive elements}

\subsubsection{Primitive elements}

Since the family $\left\{\mathbf{E}_{J}\right\}_{J \in C}$, where $C$ is the set of connected pairs of twin binary trees, are indecomposable elements of Baxter, its dual family $\left\{\mathbf{E}_{J}^{\star}\right\}_{J \in C}$ forms a basis of the Lie algebra $\mathfrak{p}^{\star}$ of the primitive elements of Baxter ${ }^{\star}$. By Proposition 6.7, the Lie algebra $\mathfrak{p}^{\star}$ is free. 


\subsubsection{Totally primitive elements}

An element $x$ of a bidendriform bialgebra is totally primitive if $\Delta_{\prec}(x)=0=\Delta_{\succ}(x)$.

Following [9], the generating series $B_{T}(z)$ of the totally primitive elements of Baxter is $B_{T}(z)=$ $\frac{B(z)-1}{B(z)^{2}}$. First dimensions of totally primitive elements of Baxter are $0,1,0,1,4,19,96,511,2832$, $16215,95374,573837$. Here follows a basis of the totally primitive elements of Baxter of order 1, 3 and 4 :

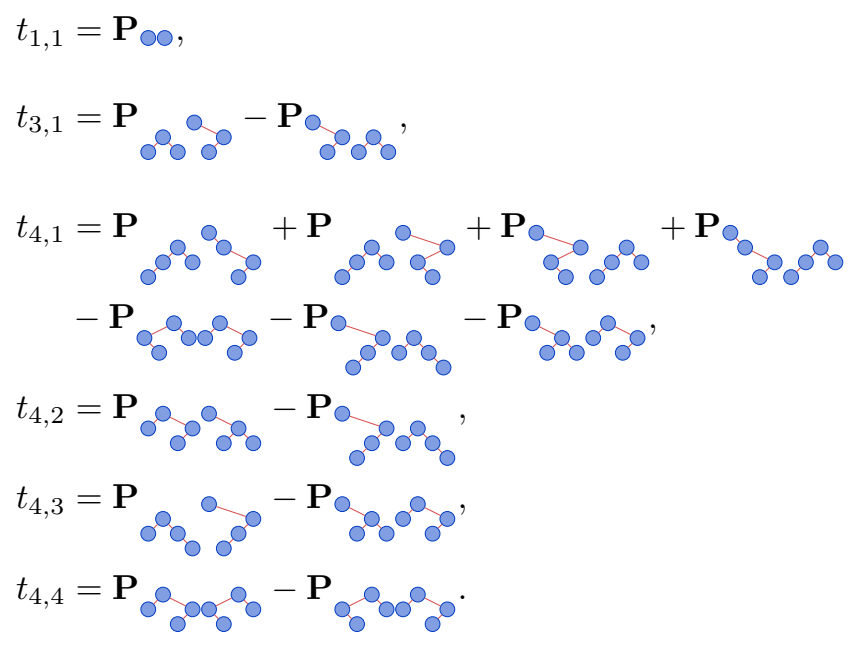

Baxter is free as dendriform algebra on its totally primitive elements.

\section{References}

[1] E. Ackerman, G. Barequet, and R. Y. Pinter. On the Number of Rectangular Partitions. Proc. 15th ACM-SIAM Symp. on Discrete Algorithms, 2004.

[2] A. Aho and J. Ullman. Foundations of Computer Science. W. H. Freeman, 1994.

[3] E. Babson and E. Steingrímsson. Generalized permutation patterns and a classification of the Mahonian statistic. Séminaire Lotharingien de Combinatoire, B44b, 2000.

[4] G. Baxter. On fixed points of the composite of commuting functions. Proceedings of the American Mathematical Society, 15:851-855, 1964.

[5] N. Bonichon, M. Bousquet-Mélou, and É. Fusy. Baxter permutations and plane bipolar orientations. Electronic Notes in Discrete Mathematics, 31:69-74, 2008.

[6] Ivan Chajda and Václav Snásel. Congruences in Ordered Sets. Mathematica Bohemica, 123:95-100, 1998.

[7] G. Duchamp, F. Hivert, and J.-Y. Thibon. Noncommutative Symmetric Functions VI: Free QuasiSymmetric Functions and Related Algebras. International Journal of Algebra and Computation, 12:671-717, 2002.

[8] S. Dulucq and O. Guibert. Mots de piles, tableaux standards et permutations de Baxter. Formal Power Series and Algebraic Combinatorics, 1994. 
[9] L. Foissy. Bidendriform bialgebras, trees, and free quasi-symmetric functions. arXiv:math/0505207v1 [math.RA], 2005.

[10] I.M. Gelfand, D. Krob, A. Lascoux, B. Leclerc, V.S. Retakh, and J.-Y. Thibon. Noncommutative symmetric functions I. arXiv: hep-th/9407124v1, 1994.

[11] F. Hivert, J.-C. Novelli, and J.-Y. Thibon. An analogue of the plactic monoid for binary search trees. Comptes Rendus Mathematique, 335, Number 7:577-580(4), 2002.

[12] F. Hivert, J.-C. Novelli, and J.-Y. Thibon. The Algebra of Binary Search Trees. Theoretical Computer Science, 339, Issue 1:129-165, 2005.

[13] F. Hivert and J. Nzeutchap. Dual graded graphs in combinatorial Hopf algebras. 2007.

[14] D. Knuth. The Art of Computer Programming, Generating All Trees, History of Combinatorial Generation, volume 4. Addison-Wesley, 2006.

[15] A. Lascoux and M.-P. Schützenberger. Le monoïde plaxique. Noncommutative Structures in Algebra and Geometric Combinatorics, pages 129-156, 1981.

[16] S Law and N Reading. The Hopf algebra of diagonal rectangulations. arXiv:1011.2118v1 [math.CO], 2010.

[17] J.-L. Loday. Dialgebras. Lecture Notes in Math., 1763:7-66, 2001.

[18] J.-L. Loday and M. O. Ronco. Hopf Algebra of the Planar Binary Trees. Advances in Mathematics, 139:293-309, 1998.

[19] C. Malvenuto and C. Reutenauer. Duality between quasi-symmetric functions and Solomon descent algebra. J. Algebra, 177:967-982, 1995.

[20] J.-C. Novelli. On the hypoplactic monoid. Discrete Mathematics, 217, Issues 1-3:315-336, 1998.

[21] S. Poirier and C. Reutenauer. Algèbres de Hopf de tableaux. Ann. Sci. Math. Québec, 19:79-90, 1995.

[22] N. Reading. Lattice congruences, fans and Hopf algebras. Journal of Combinatorial Theory Series A, 110:237-273, 2005.

[23] M. Rey. Algebraic constructions on set partitions. Formal Power Series and Algebraic Combinatorics, 2007.

[24] N. J. A. Sloane. The on-line encyclopedia of integer sequences. http://www . research . att. $\mathrm{com} / \sim \mathrm{njas} / \mathrm{sequences/}$

[25] W. A. Stein et al. Sage Mathematics Software (Version 4.5.3). The Sage Development Team, 2010. http://www.sagemath.org.

[26] X. Viennot. Up-down sequences of permutations, paths and canopy of binary trees, 2004. invited talk at the Lascouxfest, 52nd SLC, Otrott. 